Supplement of Atmos. Chem. Phys., 15, 10597-10618, 2015

http://www.atmos-chem-phys.net/15/10597/2015/

doi:10.5194/acp-15-10597-2015-supplement

(C) Author(s) 2015. CC Attribution 3.0 License.

(c) (i)

\title{
A global aerosol classification algorithm incorporating multiple satellite data sets of aerosol and trace gas abundances
}

M. J. M. Penning de Vries et al.

Correspondence to: M. J. M. Penning de Vries (marloes.penningdevries@mpic.de)

The copyright of individual parts of the supplement might differ from the CC-BY 3.0 licence. 


\section{Subdivision of urban/industrial source}

The additional information on urban/industrial (URB) sources that can be gained by displaying the enhancements in trace gases other than $\mathrm{NO}_{2}$ is introduced in the manuscript (Sect. 4.2). In Fig. 8, the results of this analysis were shown for summer 2007-2011; here we present all four seasons.
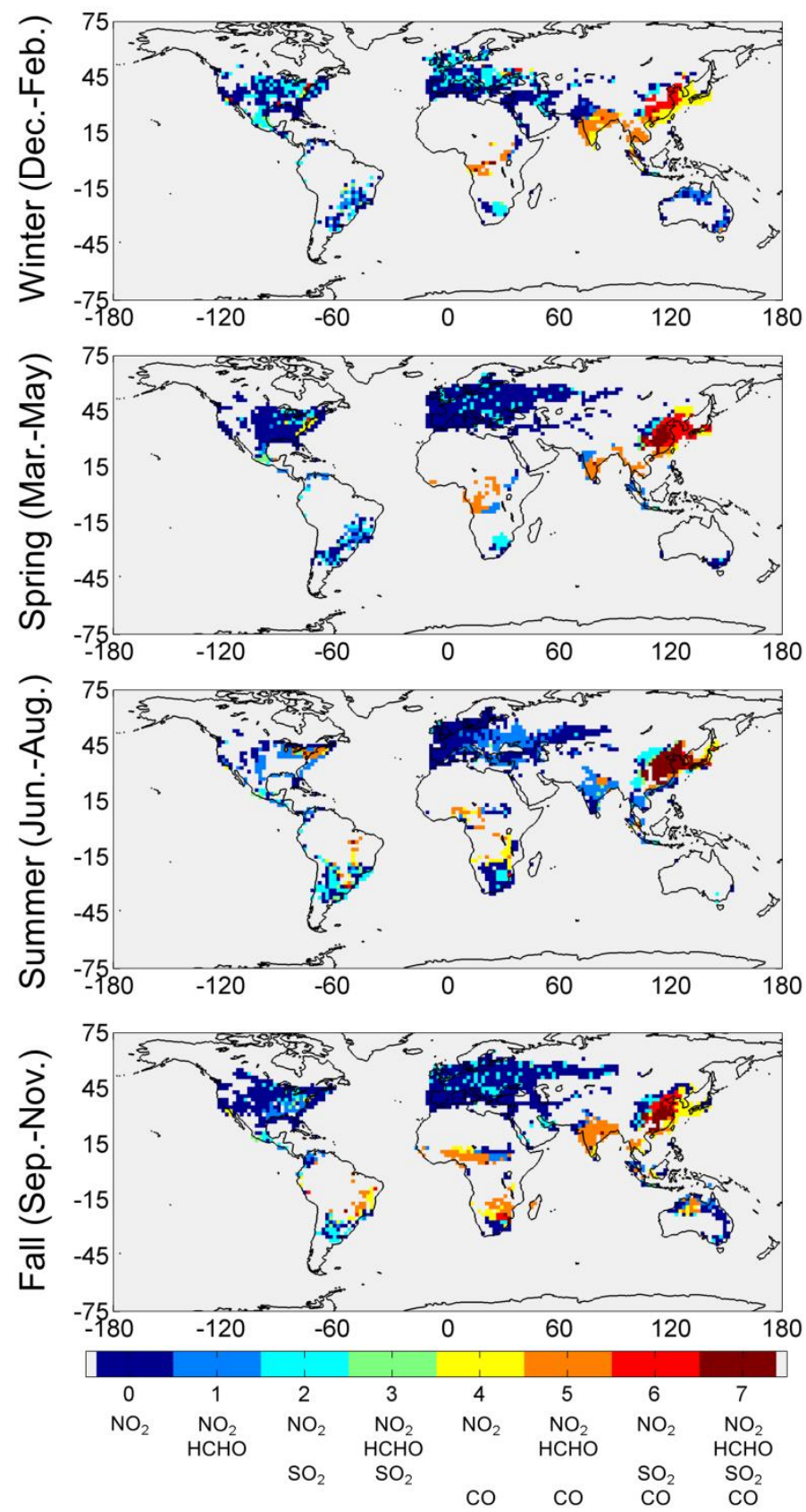

Figure S1. Trace gas composition for grid boxes with URB source type. Data are from (top to bottom): December-February, March-May, June-August, and September-November 2007-2011. The presence of enhanced trace gas columns (in addition to $\mathrm{NO}_{2}$ ) is indicated by 1,2 , or 4 for $\mathrm{HCHO}, \mathrm{SO}_{2}$, and $\mathrm{CO}$, respectively: 1 thus indicates enhanced $\mathrm{NO}_{2}$ and $\mathrm{HCHO}, 2$ enhanced $\mathrm{NO}_{2}$ and $\mathrm{SO}_{2}, 3$ enhanced $\mathrm{NO}_{2}$ and $\mathrm{HCHO}$ and $\mathrm{SO}_{2}$, etc. Gray areas are not dominated by URB. Note that the third panel from the top is identical to Fig. 8 in the manuscript. 


\section{Sensitivity studies}

\subsection{Cloud fraction}

To investigate the dependence of GACA results to cloud cover, the algorithm was run with monthly mean trace gas data to which different cloud filters had been applied: effective cloud fraction (CF) up to $5 \%, 20 \%, 40 \%$, and $100 \%$ were tested, as well as CF $>40 \%$. The cloud filter was only applied to the short-lived trace gases $\mathrm{NO}_{2}, \mathrm{HCHO}$, and $\mathrm{SO}_{2}$, and to UVAI.

There is a clear shift to more neutral aerosols at the cost of non-absorbing aerosols with increasing CF, which is due to the influence of clouds on UVAI. In addition, for the strictest CF criterion (upper plot), much of the large absorbing particles over the equatorial Atlantic Ocean are lost. This is attributed to mis-classification of desert dust by CF algorithm, FRESCO.
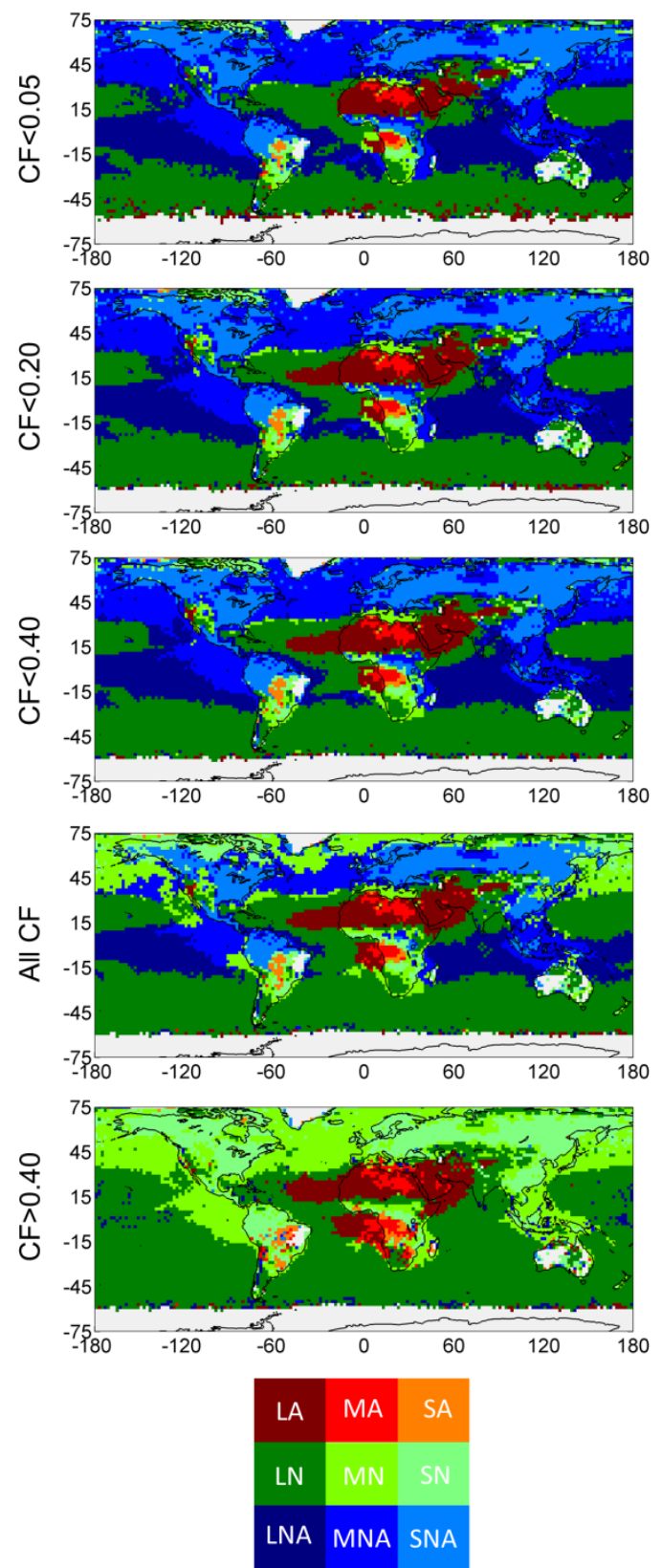

Figure S2. Global aerosol type distribution according to GACA-type for different cloud filter settings. Data are from June-August 2007-2011; the second panel from the top is identical to the third panel of Fig. 4 in the manuscript (except for the resolution). 
The source type maps are hardly influenced by changes in cloud filter settings; in the upper panel $(\mathrm{CF}<5 \%)$ the plume of desert dust over ocean is lost (see above) and in the lowermost panel the strong decrease in SNA aerosols leads to the disappearance of BIO. Otherwise, the patterns remain unchanged.
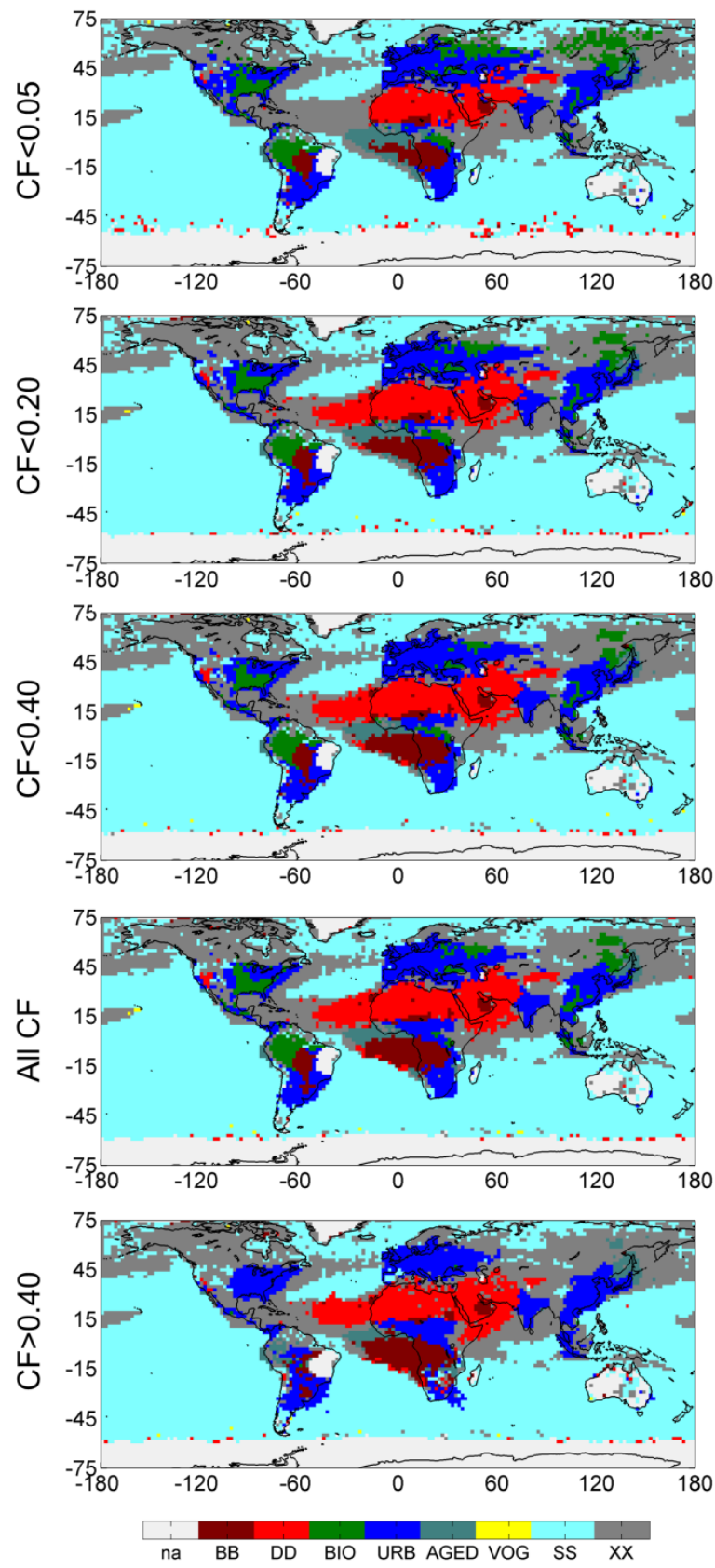

Figure S3. Global aerosol source distribution according to GACA-source for different cloud filter settings. Data are from June-August 2007-2011; the second panel from the top is identical to the third panel of Fig. 6 in the manuscript. 


\subsection{Outliers}

In the standard GACA set-up, outliers are discarded prior to analysis. If this step is removed from the algorithm, the resulting source maps are very similar to those from the standard run (compare Fig. S4 with Fig. 6 in the manuscript). The most apparent changes are the appearance of several VOG plumes in the spring and summer maps and the BB signal from the exceptional fire season that occurred in the summer of 2010 in Russia. In South America more grid boxes are assigned to BB in summer and fall.
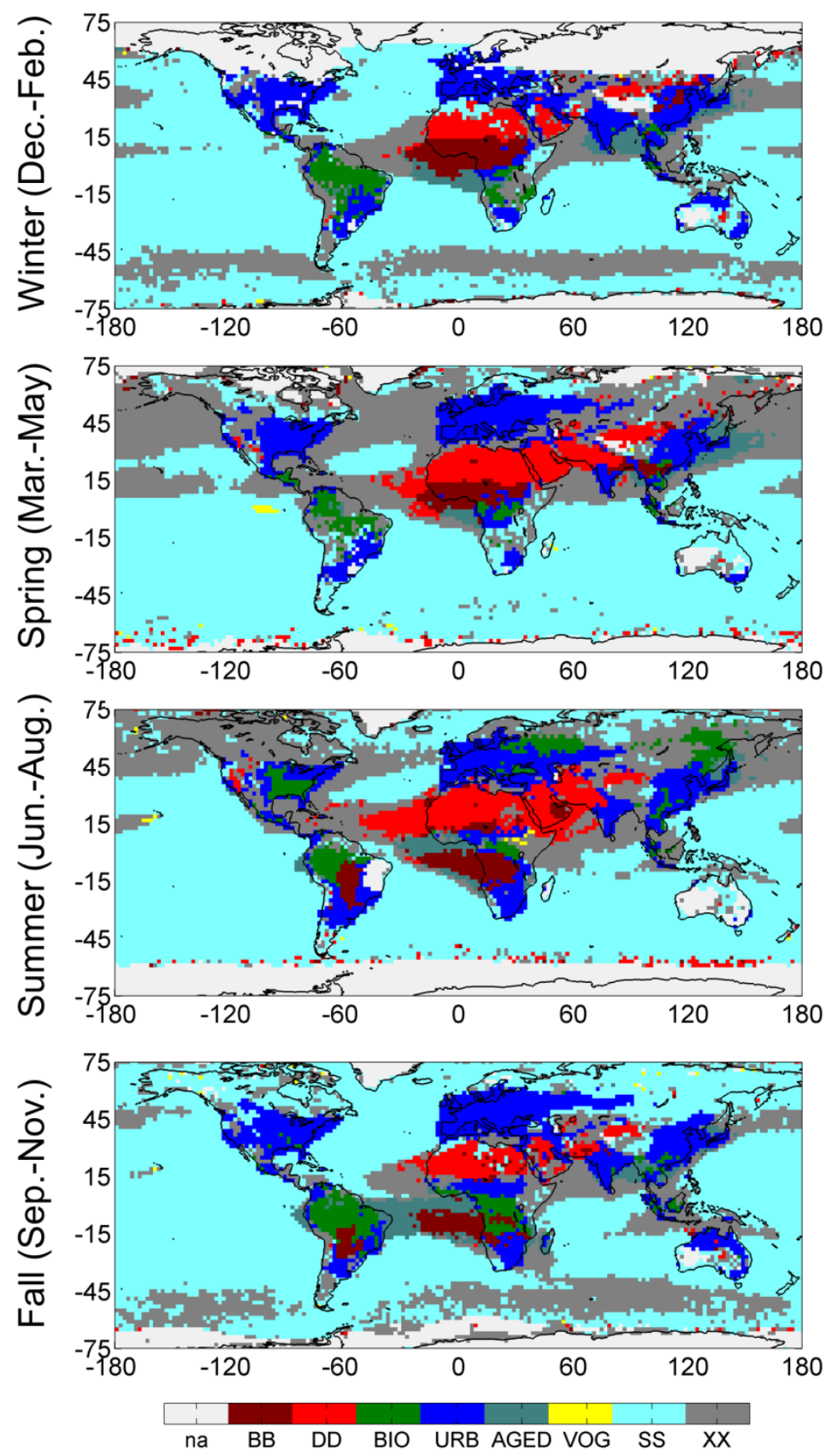

Figure S4. Global aerosol source distribution according to GACA-source without outlier removal. Data are from June-August 2007-2011. 


\section{Regional studies - data}

The following tables contain all data shown in Figs. 10-12 in the manuscript.

Table S1a. AOD assigned to each aerosol type and source by GACA (abbreviations explained in Table 1 in the manuscript) for the region in South America $\left(10-15^{\circ} \mathrm{S} / 60-65^{\circ} \mathrm{W}\right)$. Seasons: DJF: December-February, MAM: March-May, JJA: June-August, SON: September-November.

\begin{tabular}{|c|c|c|c|c|c|c|c|c|c|c|c|}
\hline \multicolumn{12}{|c|}{ GACA type } \\
\hline \multirow[t]{3}{*}{ Season } & Year & SNA & SN & SA & MNA & $\mathrm{MN}$ & MA & LNA & LN & LA & total \\
\hline & 2007 & 0.117 & 0 & 0 & 0.042 & 0 & 0 & 0.002 & 0 & 0 & 0.161 \\
\hline & 2008 & 0.092 & 0 & 0 & 0.042 & 0 & 0 & 0.005 & 0 & 0 & 0.139 \\
\hline \multirow[t]{5}{*}{ DJF } & 2009 & 0.1 & 0 & 0 & 0.06 & 0 & 0 & 0.004 & 0 & 0 & 0.164 \\
\hline & 2010 & 0.082 & 0 & 0 & 0.052 & 0 & 0 & 0.007 & 0 & 0 & 0.141 \\
\hline & 2011 & 0.122 & 0 & 0 & 0.026 & 0 & 0 & 0 & 0 & 0 & 0.148 \\
\hline & 2007 & 0.064 & 0 & 0 & 0.044 & 0 & 0 & 0.002 & 0 & 0 & 0.11 \\
\hline & 2008 & 0.054 & 0 & 0 & 0.051 & 0 & 0 & 0.003 & 0 & 0 & 0.108 \\
\hline \multirow[t]{5}{*}{ MAM } & 2009 & 0.065 & 0 & 0 & 0.048 & 0 & 0 & 0.003 & 0 & 0 & 0.116 \\
\hline & 2010 & 0.051 & 0 & 0 & 0.043 & 0 & 0 & 0.003 & 0 & 0 & 0.097 \\
\hline & 2011 & 0.077 & 0 & 0 & 0.025 & 0 & 0 & 0 & 0 & 0 & 0.102 \\
\hline & 2007 & 0.025 & 0.285 & 0 & 0 & 0 & 0 & 0 & 0 & 0 & 0.31 \\
\hline & 2008 & 0.246 & 0 & 0 & 0.017 & 0 & 0 & 0 & 0 & 0 & 0.263 \\
\hline \multirow[t]{5}{*}{ JJA } & 2009 & 0.08 & 0 & 0 & 0.003 & 0 & 0 & 0 & 0 & 0 & 0.083 \\
\hline & 2010 & 0.025 & 0.049 & 0.407 & 0 & 0.018 & 0.017 & 0 & 0 & 0 & 0.516 \\
\hline & 2011 & 0.128 & 0 & 0 & 0.006 & 0 & 0 & 0 & 0 & 0 & 0.134 \\
\hline & 2007 & 0.144 & 0.216 & 0.396 & 0 & 0 & 0.394 & 0 & 0 & 0 & 1.15 \\
\hline & 2008 & 0.341 & 0.012 & 0 & 0 & 0 & 0 & 0 & 0 & 0 & 0.353 \\
\hline \multirow[t]{3}{*}{ SON } & 2009 & 0.235 & 0 & 0 & 0 & 0 & 0 & 0 & 0 & 0 & 0.235 \\
\hline & 2010 & 0.235 & 0 & 0.455 & 0 & 0 & 0 & 0 & 0 & 0 & 0.69 \\
\hline & 2011 & 0.294 & 0 & 0 & 0 & 0 & 0 & 0 & 0 & 0 & 0.294 \\
\hline \multicolumn{12}{|c|}{ GACA source } \\
\hline \multirow[t]{3}{*}{ Season } & Year & na & BB & $\mathrm{DD}$ & $\mathrm{BIO}$ & URB & AGED & VOG & SS & $X X$ & total \\
\hline & 2007 & 0.002 & 0 & 0 & 0.117 & 0 & 0 & 0 & 0 & 0.042 & 0.161 \\
\hline & 2008 & 0.005 & 0 & 0 & 0.092 & 0 & 0 & 0 & 0 & 0.042 & 0.139 \\
\hline \multirow[t]{5}{*}{ DJF } & 2009 & 0.004 & 0 & 0 & 0 & 0 & 0 & 0 & 0 & 0.16 & 0.164 \\
\hline & 2010 & 0.007 & 0 & 0 & 0 & 0 & 0.082 & 0 & 0 & 0.052 & 0.141 \\
\hline & 2011 & 0 & 0 & 0 & 0.122 & 0 & 0 & 0 & 0 & 0.026 & 0.148 \\
\hline & 2007 & 0.002 & 0 & 0 & 0.064 & 0 & 0 & 0 & 0.044 & 0 & 0.11 \\
\hline & 2008 & 0.003 & 0 & 0 & 0 & 0 & 0 & 0 & 0.051 & 0.054 & 0.108 \\
\hline \multirow[t]{5}{*}{ MAM } & 2009 & 0.003 & 0 & 0 & 0 & 0 & 0 & 0 & 0.048 & 0.065 & 0.116 \\
\hline & 2010 & 0.003 & 0 & 0 & 0 & 0 & 0 & 0 & 0.043 & 0.051 & 0.097 \\
\hline & 2011 & 0 & 0 & 0 & 0.077 & 0 & 0 & 0 & 0.025 & 0 & 0.102 \\
\hline & 2007 & 0 & 0 & 0 & 0.025 & 0.285 & 0 & 0 & 0 & 0 & 0.31 \\
\hline & 2008 & 0.017 & 0 & 0 & 0.246 & 0 & 0 & 0 & 0 & 0 & 0.263 \\
\hline \multirow[t]{5}{*}{ JJA } & 2009 & 0.003 & 0 & 0 & 0.08 & 0 & 0 & 0 & 0 & 0 & 0.083 \\
\hline & 2010 & 0.084 & 0.407 & 0 & 0.025 & 0 & 0 & 0 & 0 & 0 & 0.516 \\
\hline & 2011 & 0.006 & 0 & 0 & 0.128 & 0 & 0 & 0 & 0 & 0 & 0.134 \\
\hline & 2007 & 0 & 0.79 & 0 & 0.144 & 0.216 & 0 & 0 & 0 & 0 & 1.15 \\
\hline & 2008 & 0.012 & 0 & 0 & 0.341 & 0 & 0 & 0 & 0 & 0 & 0.353 \\
\hline \multirow[t]{3}{*}{ SON } & 2009 & 0 & 0 & 0 & 0.235 & 0 & 0 & 0 & 0 & 0 & 0.235 \\
\hline & 2010 & 0 & 0.455 & 0 & 0.235 & 0 & 0 & 0 & 0 & 0 & 0.69 \\
\hline & 2011 & 0 & 0 & 0 & 0.294 & 0 & 0 & 0 & 0 & 0 & 0.294 \\
\hline
\end{tabular}


Table S1b. AOD assigned to each aerosol component by MACC (abbreviations explained in Table 1 in the manuscript) for the region in South America (10-15 $\left.\mathrm{S} / 60-65^{\circ} \mathrm{W}\right)$. Seasons: DJF: DecemberFebruary, MAM: March-May, JJA: June-August, SON: September-November.

\begin{tabular}{|l|l|l|r|r|r|r|r|}
\hline Season & Year & BC & \multicolumn{1}{l|}{ DD } & \multicolumn{1}{l|}{ OC } & \multicolumn{1}{l|}{ SO4 } & \multicolumn{1}{l|}{ SS } & \multicolumn{1}{l|}{ total } \\
\hline & 2007 & 0.002 & 0.04 & 0.087 & 0.024 & 0.033 & 0.186 \\
\hline & 2008 & 0.002 & 0.045 & 0.104 & 0.028 & 0.041 & 0.22 \\
\hline DJF & 2009 & 0.003 & 0.036 & 0.114 & 0.03 & 0.043 & 0.226 \\
\hline & 2010 & 0.002 & 0.031 & 0.128 & 0.025 & 0.031 & 0.217 \\
\hline & 2011 & 0.006 & 0.033 & 0.121 & 0.057 & 0.058 & 0.274 \\
\hline & 2007 & 0.003 & 0.021 & 0.077 & 0.02 & 0.019 & 0.141 \\
\hline & 2008 & 0.003 & 0.031 & 0.081 & 0.024 & 0.027 & 0.167 \\
\hline MAM & 2009 & 0.002 & 0.021 & 0.079 & 0.02 & 0.023 & 0.146 \\
\hline & 2010 & 0.002 & 0.02 & 0.093 & 0.019 & 0.019 & 0.153 \\
\hline & 2011 & 0.003 & 0.026 & 0.08 & 0.027 & 0.032 & 0.168 \\
\hline & 2007 & 0.031 & 0.022 & 0.237 & 0.02 & 0.018 & 0.329 \\
\hline & 2008 & 0.022 & 0.023 & 0.176 & 0.018 & 0.023 & 0.261 \\
\hline JJA & 2009 & 0.006 & 0.022 & 0.082 & 0.016 & 0.02 & 0.147 \\
\hline & 2010 & 0.019 & 0.025 & 0.208 & 0.032 & 0.022 & 0.306 \\
\hline & 2011 & 0.007 & 0.029 & 0.074 & 0.018 & 0.025 & 0.153 \\
\hline & 2007 & 0.094 & 0.048 & 0.676 & 0.062 & 0.076 & 0.955 \\
\hline & 2008 & 0.025 & 0.042 & 0.242 & 0.039 & 0.06 & 0.407 \\
\hline SON & 2009 & 0.014 & 0.045 & 0.171 & 0.035 & 0.054 & 0.319 \\
\hline & 2010 & 0.029 & 0.017 & 0.395 & 0.046 & 0.026 & 0.514 \\
\hline & 2011 & 0.022 & 0.04 & 0.206 & 0.036 & 0.057 & 0.36 \\
\hline
\end{tabular}


Table S2a. AOD assigned to each aerosol type and source by GACA (abbreviations explained in Table 1 in the manuscript) for the region in southern Africa $\left(0-5^{\circ} S / 15-20^{\circ} \mathrm{E}\right)$. Seasons: DJF:

December-February, MAM: March-May, JJA: June-August, SON: September-November.

\begin{tabular}{|c|c|c|c|c|c|c|c|c|c|c|c|}
\hline \multicolumn{12}{|c|}{ GACA type } \\
\hline \multirow[t]{3}{*}{ Season } & Year & SNA & SN & SA & MNA & $\mathrm{MN}$ & $\mathrm{MA}$ & LNA & LN & LA & total \\
\hline & 2007 & 0 & 0 & 0 & 0 & 0.13 & 0.009 & 0 & 0.04 & 0.234 & 0.413 \\
\hline & 2008 & 0.004 & 0.003 & 0 & 0.048 & 0.064 & 0.016 & 0.053 & 0.11 & 0.105 & 0.403 \\
\hline \multirow[t]{5}{*}{ DJF } & 2009 & 0.01 & 0.004 & 0 & 0.06 & 0.097 & 0.004 & 0.015 & 0.072 & 0.043 & 0.305 \\
\hline & 2010 & 0.048 & 0.004 & 0 & 0.164 & 0.119 & 0.008 & 0.004 & 0.054 & 0.007 & 0.408 \\
\hline & 2011 & 0.044 & 0 & 0 & 0.138 & 0.089 & 0 & 0.023 & 0.057 & 0.059 & 0.41 \\
\hline & 2007 & 0.083 & 0.028 & 0 & 0.062 & 0.065 & 0 & 0.005 & 0.043 & 0 & 0.286 \\
\hline & 2008 & 0.083 & 0.025 & 0 & 0.104 & 0.069 & 0 & 0 & 0 & 0 & 0.281 \\
\hline \multirow[t]{5}{*}{ MAM } & 2009 & 0.069 & 0.004 & 0 & 0.113 & 0.052 & 0 & 0 & 0.009 & 0 & 0.247 \\
\hline & 2010 & 0.054 & 0.059 & 0 & 0.126 & 0.074 & 0 & 0.006 & 0.025 & 0 & 0.344 \\
\hline & 2011 & 0.069 & 0.009 & 0 & 0.186 & 0.024 & 0 & 0.024 & 0.031 & 0 & 0.343 \\
\hline & 2007 & 0.003 & 0.029 & 0.057 & 0 & 0.145 & 0.396 & 0 & 0 & 0.023 & 0.653 \\
\hline & 2008 & 0 & 0.034 & 0.081 & 0 & 0.089 & 0.428 & 0 & 0 & 0.037 & 0.669 \\
\hline \multirow[t]{5}{*}{ JJA } & 2009 & 0.009 & 0.018 & 0.035 & 0.026 & 0.09 & 0.587 & 0 & 0 & 0.025 & 0.79 \\
\hline & 2010 & 0 & 0 & 0.053 & 0.006 & 0.122 & 0.559 & 0 & 0 & 0.031 & 0.771 \\
\hline & 2011 & 0 & 0.016 & 0.039 & 0.008 & 0.138 & 0.642 & 0 & 0 & 0 & 0.843 \\
\hline & 2007 & 0.232 & 0.07 & 0 & 0.011 & 0 & 0 & 0 & 0 & 0 & 0.313 \\
\hline & 2008 & 0.173 & 0.093 & 0.045 & 0.029 & 0 & 0 & 0 & 0 & 0 & 0.34 \\
\hline \multirow[t]{3}{*}{ SON } & 2009 & 0.141 & 0.111 & 0 & 0.08 & 0 & 0 & 0 & 0 & 0 & 0.332 \\
\hline & 2010 & 0.209 & 0.116 & 0.01 & 0 & 0 & 0 & 0 & 0 & 0 & 0.335 \\
\hline & 2011 & 0.309 & 0.131 & 0.063 & 0 & 0 & 0 & 0 & 0 & 0 & 0.503 \\
\hline \multicolumn{12}{|c|}{ GACA source } \\
\hline \multirow[t]{3}{*}{ Season } & Year & na & $\mathrm{BB}$ & $\mathrm{DD}$ & $\mathrm{BIO}$ & URB & AGED & VOG & SS & $X X$ & total \\
\hline & 2007 & 0.009 & 0.234 & 0 & 0 & 0.17 & 0 & 0 & 0 & 0 & 0.413 \\
\hline & 2008 & 0.023 & 0.105 & 0 & 0 & 0.276 & 0 & 0 & 0 & 0 & 0.404 \\
\hline \multirow[t]{5}{*}{ DJF } & 2009 & 0.032 & 0.043 & 0 & 0 & 0.229 & 0 & 0 & 0 & 0 & 0.304 \\
\hline & 2010 & 0.023 & 0 & 0 & 0.048 & 0.337 & 0 & 0 & 0 & 0 & 0.408 \\
\hline & 2011 & 0.023 & 0.059 & 0 & 0.044 & 0.284 & 0 & 0 & 0 & 0 & 0.41 \\
\hline & 2007 & 0.005 & 0 & 0 & 0.083 & 0.17 & 0 & 0 & 0 & 0.028 & 0.286 \\
\hline & 2008 & 0 & 0.025 & 0 & 0.083 & 0.173 & 0 & 0 & 0 & 0 & 0.281 \\
\hline \multirow[t]{5}{*}{ MAM } & 2009 & 0.013 & 0 & 0 & 0.069 & 0.165 & 0 & 0 & 0 & 0 & 0.247 \\
\hline & 2010 & 0.031 & 0 & 0 & 0.054 & 0.259 & 0 & 0 & 0 & 0 & 0.344 \\
\hline & 2011 & 0.034 & 0 & 0 & 0.069 & 0.217 & 0 & 0 & 0 & 0.024 & 0.344 \\
\hline & 2007 & 0.026 & 0.482 & 0 & 0 & 0.145 & 0 & 0 & 0 & 0 & 0.653 \\
\hline & 2008 & 0.037 & 0.509 & 0 & 0 & 0.089 & 0 & 0 & 0 & 0.034 & 0.669 \\
\hline \multirow[t]{5}{*}{ JJA } & 2009 & 0.112 & 0.587 & 0 & 0 & 0.09 & 0 & 0 & 0 & 0 & 0.789 \\
\hline & 2010 & 0.037 & 0.611 & 0 & 0 & 0.122 & 0 & 0 & 0 & 0 & 0.77 \\
\hline & 2011 & 0.064 & 0.642 & 0 & 0 & 0.138 & 0 & 0 & 0 & 0 & 0.844 \\
\hline & 2007 & 0.011 & 0 & 0 & 0.232 & 0.07 & 0 & 0 & 0 & 0 & 0.313 \\
\hline & 2008 & 0 & 0.045 & 0 & 0.173 & 0.122 & 0 & 0 & 0 & 0 & 0.34 \\
\hline \multirow[t]{3}{*}{ SON } & 2009 & 0 & 0 & 0 & 0.141 & 0.191 & 0 & 0 & 0 & 0 & 0.332 \\
\hline & 2010 & 0.01 & 0 & 0 & 0.209 & 0.116 & 0 & 0 & 0 & 0 & 0.335 \\
\hline & 2011 & 0.063 & 0 & 0 & 0.309 & 0.131 & 0 & 0 & 0 & 0 & 0.503 \\
\hline
\end{tabular}


Table S2b. AOD assigned to each aerosol component by MACC(abbreviations explained in Table 1 in the manuscript) for the region in southern Africa $\left(0-5^{\circ} \mathrm{S} / 15-20^{\circ} \mathrm{E}\right)$. Seasons: DJF: December-February, MAM: March-May, JJA: June-August, SON: September-November.

\begin{tabular}{|l|l|r|r|r|r|r|r|}
\hline Season & Year & BC & \multicolumn{1}{l|}{ DD } & \multicolumn{1}{l|}{ OC } & \multicolumn{1}{l|}{ SO4 } & \multicolumn{1}{l|}{ SS } & \multicolumn{1}{l|}{ total } \\
\hline & 2007 & 0.021 & 0.156 & 0.183 & 0.042 & 0.025 & 0.427 \\
\hline & 2008 & 0.017 & 0.129 & 0.158 & 0.042 & 0.027 & 0.373 \\
\hline DJF & 2009 & 0.016 & 0.101 & 0.147 & 0.039 & 0.03 & 0.332 \\
\hline & 2010 & 0.017 & 0.119 & 0.167 & 0.039 & 0.027 & 0.369 \\
\hline & 2011 & 0.021 & 0.134 & 0.195 & 0.048 & 0.027 & 0.424 \\
\hline & 2007 & 0.012 & 0.076 & 0.129 & 0.039 & 0.039 & 0.296 \\
\hline & 2008 & 0.012 & 0.066 & 0.119 & 0.044 & 0.045 & 0.286 \\
\hline MAM & 2009 & 0.011 & 0.072 & 0.12 & 0.037 & 0.041 & 0.281 \\
\hline & 2010 & 0.013 & 0.076 & 0.146 & 0.04 & 0.04 & 0.316 \\
\hline & 2011 & 0.014 & 0.098 & 0.141 & 0.05 & 0.051 & 0.354 \\
\hline & 2007 & 0.057 & 0.04 & 0.438 & 0.049 & 0.05 & 0.634 \\
\hline & 2008 & 0.062 & 0.065 & 0.47 & 0.056 & 0.073 & 0.727 \\
\hline JJA & 2009 & 0.076 & 0.076 & 0.486 & 0.062 & 0.074 & 0.775 \\
\hline & 2010 & 0.072 & 0.061 & 0.506 & 0.064 & 0.065 & 0.767 \\
\hline & 2011 & 0.077 & 0.089 & 0.499 & 0.076 & 0.087 & 0.829 \\
\hline & 2007 & 0.02 & 0.038 & 0.174 & 0.036 & 0.052 & 0.32 \\
\hline & 2008 & 0.024 & 0.06 & 0.198 & 0.039 & 0.053 & 0.374 \\
\hline SON & 2009 & 0.024 & 0.047 & 0.189 & 0.041 & 0.048 & 0.35 \\
\hline & 2010 & 0.02 & 0.04 & 0.197 & 0.043 & 0.048 & 0.349 \\
\hline & 2011 & 0.024 & 0.074 & 0.209 & 0.044 & 0.066 & 0.417 \\
\hline
\end{tabular}


Table S3a. AOD assigned to each aerosol type and source by GACA (abbreviations explained in Table 1 in the manuscript) for the region in Southeast USA $\left(30-35^{\circ} \mathrm{N} / 80-85^{\circ} \mathrm{W}\right)$. Seasons: DJF: December-February, MAM: March-May, JJA: June-August, SON: September-November.

\begin{tabular}{|c|c|c|c|c|c|c|c|c|c|c|c|}
\hline \multicolumn{12}{|c|}{ GACA type } \\
\hline \multirow[t]{3}{*}{ Season } & Year & SNA & SN & SA & MNA & $\mathrm{MN}$ & MA & LNA & LN & LA & total \\
\hline & 2007 & 0.018 & 0.022 & 0 & 0.024 & 0.015 & 0 & 0 & 0 & 0 & 0.079 \\
\hline & 2008 & 0.019 & 0 & 0 & 0.052 & 0.008 & 0 & 0 & 0 & 0 & 0.079 \\
\hline \multirow[t]{5}{*}{ DJF } & 2009 & 0.027 & 0.018 & 0 & 0.019 & 0.004 & 0 & 0 & 0 & 0 & 0.068 \\
\hline & 2010 & 0.026 & 0 & 0 & 0.038 & 0 & 0 & 0 & 0 & 0 & 0.064 \\
\hline & 2011 & 0.039 & 0 & 0 & 0.028 & 0 & 0 & 0 & 0 & 0 & 0.067 \\
\hline & 2007 & 0.145 & 0.009 & 0 & 0.002 & 0 & 0 & 0 & 0 & 0 & 0.156 \\
\hline & 2008 & 0.091 & 0 & 0 & 0.021 & 0 & 0 & 0 & 0 & 0 & 0.112 \\
\hline \multirow[t]{5}{*}{ MAM } & 2009 & 0.089 & 0 & 0 & 0.029 & 0 & 0 & 0 & 0 & 0 & 0.118 \\
\hline & 2010 & 0.073 & 0 & 0 & 0.033 & 0 & 0 & 0 & 0 & 0 & 0.106 \\
\hline & 2011 & 0.128 & 0 & 0 & 0.011 & 0 & 0 & 0 & 0 & 0 & 0.139 \\
\hline & 2007 & 0.361 & 0 & 0 & 0.022 & 0 & 0 & 0 & 0 & 0 & 0.383 \\
\hline & 2008 & 0.266 & 0 & 0 & 0 & 0 & 0 & 0 & 0 & 0 & 0.266 \\
\hline \multirow[t]{5}{*}{ JJA } & 2009 & 0.211 & 0 & 0 & 0.017 & 0 & 0 & 0 & 0 & 0 & 0.228 \\
\hline & 2010 & 0.208 & 0 & 0 & 0 & 0 & 0 & 0 & 0 & 0 & 0.208 \\
\hline & 2011 & 0.336 & 0 & 0 & 0 & 0 & 0 & 0 & 0 & 0 & 0.336 \\
\hline & 2007 & 0.105 & 0 & 0 & 0.014 & 0 & 0 & 0 & 0 & 0 & 0.119 \\
\hline & 2008 & 0.098 & 0 & 0 & 0.01 & 0 & 0 & 0 & 0 & 0 & 0.108 \\
\hline \multirow[t]{3}{*}{ SON } & 2009 & 0.083 & 0 & 0 & 0.005 & 0 & 0 & 0 & 0 & 0 & 0.088 \\
\hline & 2010 & 0.101 & 0 & 0 & 0.01 & 0 & 0 & 0 & 0 & 0 & 0.111 \\
\hline & 2011 & 0.139 & 0 & 0 & 0.006 & 0 & 0 & 0 & 0 & 0 & 0.145 \\
\hline \multicolumn{12}{|c|}{ GACA source } \\
\hline \multirow[t]{3}{*}{ Season } & Year & na & $\mathrm{BB}$ & $\mathrm{DD}$ & $\mathrm{BIO}$ & URB & AGED & VOG & SS & $X X$ & total \\
\hline & 2007 & 0.039 & 0 & 0 & 0 & 0.04 & 0 & 0 & 0 & 0 & 0.079 \\
\hline & 2008 & 0.027 & 0 & 0 & 0 & 0.052 & 0 & 0 & 0 & 0 & 0.079 \\
\hline \multirow[t]{5}{*}{ DJF } & 2009 & 0.041 & 0 & 0 & 0 & 0.027 & 0 & 0 & 0 & 0 & 0.068 \\
\hline & 2010 & 0.064 & 0 & 0 & 0 & 0 & 0 & 0 & 0 & 0 & 0.064 \\
\hline & 2011 & 0 & 0 & 0 & 0 & 0.039 & 0 & 0 & 0.028 & 0 & 0.067 \\
\hline & 2007 & 0.012 & 0 & 0 & 0 & 0.145 & 0 & 0 & 0 & 0 & 0.157 \\
\hline & 2008 & 0 & 0 & 0 & 0 & 0.112 & 0 & 0 & 0 & 0 & 0.112 \\
\hline \multirow[t]{5}{*}{ MAM } & 2009 & 0 & 0 & 0 & 0 & 0.118 & 0 & 0 & 0 & 0 & 0.118 \\
\hline & 2010 & 0 & 0 & 0 & 0 & 0.107 & 0 & 0 & 0 & 0 & 0.107 \\
\hline & 2011 & 0 & 0 & 0 & 0 & 0.14 & 0 & 0 & 0 & 0 & 0.14 \\
\hline & 2007 & 0 & 0 & 0 & 0.361 & 0.022 & 0 & 0 & 0 & 0 & 0.383 \\
\hline & 2008 & 0 & 0 & 0 & 0.266 & 0 & 0 & 0 & 0 & 0 & 0.266 \\
\hline \multirow[t]{5}{*}{ JJA } & 2009 & 0 & 0 & 0 & 0.211 & 0.017 & 0 & 0 & 0 & 0 & 0.228 \\
\hline & 2010 & 0 & 0 & 0 & 0.208 & 0 & 0 & 0 & 0 & 0 & 0.208 \\
\hline & 2011 & 0 & 0 & 0 & 0.336 & 0 & 0 & 0 & 0 & 0 & 0.336 \\
\hline & 2007 & 0 & 0 & 0 & 0 & 0.12 & 0 & 0 & 0 & 0 & 0.12 \\
\hline & 2008 & 0.01 & 0 & 0 & 0 & 0.098 & 0 & 0 & 0 & 0 & 0.108 \\
\hline \multirow[t]{3}{*}{ SON } & 2009 & 0.005 & 0 & 0 & 0 & 0.083 & 0 & 0 & 0 & 0 & 0.088 \\
\hline & 2010 & 0.01 & 0 & 0 & 0.101 & 0 & 0 & 0 & 0 & 0 & 0.111 \\
\hline & 2011 & 0.006 & 0 & 0 & 0.139 & 0 & 0 & 0 & 0 & 0 & 0.145 \\
\hline
\end{tabular}


Table S3b. AOD assigned to each aerosol component by MACC (abbreviations explained in Table 1 in

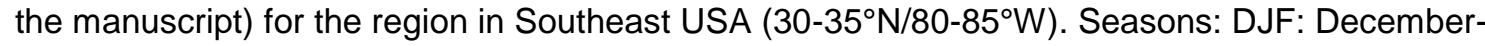
February, MAM: March-May, JJA: June-August, SON: September-November.

\begin{tabular}{|l|r|r|r|r|r|r|r|}
\hline Season & Year & BC & \multicolumn{1}{l|}{ DD } & \multicolumn{1}{l|}{ OC } & \multicolumn{1}{l|}{ SO4 } & \multicolumn{1}{l|}{ SS } & \multicolumn{1}{l|}{ total } \\
\hline & 2007 & 0.006 & 0.021 & 0.013 & 0.07 & 0.03 & 0.141 \\
\hline & 2008 & 0.009 & 0.032 & 0.016 & 0.087 & 0.036 & 0.18 \\
\hline DJF & 2009 & 0.006 & 0.027 & 0.02 & 0.094 & 0.049 & 0.196 \\
\hline & 2010 & 0.006 & 0.017 & 0.011 & 0.066 & 0.018 & 0.119 \\
\hline & 2011 & 0.008 & 0.028 & 0.038 & 0.09 & 0.034 & 0.198 \\
\hline & 2007 & 0.01 & 0.039 & 0.035 & 0.147 & 0.025 & 0.256 \\
\hline & 2008 & 0.008 & 0.049 & 0.027 & 0.124 & 0.031 & 0.239 \\
\hline MAM & 2009 & 0.007 & 0.043 & 0.031 & 0.121 & 0.038 & 0.24 \\
\hline & 2010 & 0.006 & 0.036 & 0.03 & 0.122 & 0.022 & 0.216 \\
\hline & 2011 & 0.012 & 0.055 & 0.047 & 0.126 & 0.03 & 0.268 \\
\hline & 2007 & 0.011 & 0.064 & 0.067 & 0.248 & 0.025 & 0.416 \\
\hline & 2008 & 0.009 & 0.078 & 0.053 & 0.176 & 0.027 & 0.342 \\
\hline JJA & 2009 & 0.007 & 0.067 & 0.05 & 0.15 & 0.022 & 0.296 \\
\hline & 2010 & 0.005 & 0.05 & 0.054 & 0.168 & 0.026 & 0.303 \\
\hline & 2011 & 0.011 & 0.075 & 0.083 & 0.196 & 0.027 & 0.392 \\
\hline & 2007 & 0.006 & 0.031 & 0.018 & 0.089 & 0.026 & 0.17 \\
\hline & 2008 & 0.004 & 0.035 & 0.015 & 0.077 & 0.027 & 0.158 \\
\hline SON & 2009 & 0.005 & 0.031 & 0.022 & 0.093 & 0.023 & 0.173 \\
\hline & 2010 & 0.005 & 0.025 & 0.02 & 0.083 & 0.016 & 0.148 \\
\hline & 2011 & 0.006 & 0.035 & 0.022 & 0.088 & 0.027 & 0.177 \\
\hline
\end{tabular}


Table S4a. AOD assigned to each aerosol type and source by GACA (abbreviations explained in Table 1 in the manuscript) for the region in Northwest Europe $\left(48-53^{\circ} \mathrm{N} / 3-8^{\circ} \mathrm{E}\right)$. Seasons: DJF: December-February, MAM: March-May, JJA: June-August, SON: September-November.

\begin{tabular}{|c|c|c|c|c|c|c|c|c|c|c|c|}
\hline \multicolumn{12}{|c|}{ GACA type } \\
\hline \multirow[t]{3}{*}{ Season } & Year & SNA & SN & SA & MNA & $\mathrm{MN}$ & MA & LNA & LN & LA & total \\
\hline & 2007 & 0 & 0.019 & 0 & 0 & 0.092 & 0 & 0 & 0 & 0 & 0.111 \\
\hline & 2008 & 0 & 0.013 & 0 & 0 & 0.088 & 0 & 0 & 0.035 & 0 & 0.136 \\
\hline \multirow[t]{5}{*}{ DJF } & 2009 & 0 & 0 & 0 & 0.007 & 0.06 & 0 & 0 & 0.065 & 0.005 & 0.137 \\
\hline & 2010 & 0 & 0.011 & 0 & 0 & 0.03 & 0 & 0.069 & 0.029 & 0 & 0.139 \\
\hline & 2011 & 0 & 0.031 & 0 & 0.022 & 0.023 & 0 & 0.006 & 0.019 & 0 & 0.101 \\
\hline & 2007 & 0.142 & 0.015 & 0 & 0.049 & 0 & 0 & 0 & 0 & 0 & 0.206 \\
\hline & 2008 & 0.105 & 0 & 0 & 0.05 & 0.006 & 0 & 0 & 0 & 0 & 0.161 \\
\hline \multirow[t]{5}{*}{ MAM } & 2009 & 0.142 & 0.004 & 0 & 0.049 & 0.006 & 0 & 0 & 0 & 0 & 0.201 \\
\hline & 2010 & 0.084 & 0.001 & 0 & 0.064 & 0 & 0 & 0.015 & 0 & 0 & 0.164 \\
\hline & 2011 & 0.123 & 0 & 0 & 0.071 & 0 & 0 & 0 & 0 & 0 & 0.194 \\
\hline & 2007 & 0.135 & 0 & 0 & 0.082 & 0 & 0 & 0.003 & 0 & 0 & 0.22 \\
\hline & 2008 & 0.11 & 0 & 0 & 0.06 & 0 & 0 & 0.007 & 0 & 0 & 0.177 \\
\hline \multirow[t]{5}{*}{ JJA } & 2009 & 0.128 & 0 & 0 & 0.06 & 0 & 0 & 0.003 & 0 & 0 & 0.191 \\
\hline & 2010 & 0.082 & 0 & 0 & 0.068 & 0 & 0 & 0.004 & 0 & 0 & 0.154 \\
\hline & 2011 & 0.112 & 0 & 0 & 0.051 & 0 & 0 & 0.009 & 0 & 0 & 0.172 \\
\hline & 2007 & 0.083 & 0.011 & 0 & 0.018 & 0.013 & 0 & 0.003 & 0 & 0 & 0.128 \\
\hline & 2008 & 0.06 & 0.004 & 0 & 0.042 & 0.02 & 0 & 0.008 & 0.011 & 0 & 0.145 \\
\hline \multirow[t]{3}{*}{ SON } & 2009 & 0.072 & 0.014 & 0 & 0.029 & 0.007 & 0 & 0.012 & 0.01 & 0 & 0.144 \\
\hline & 2010 & 0.04 & 0.003 & 0 & 0.035 & 0.001 & 0 & 0.003 & 0.002 & 0 & 0.084 \\
\hline & 2011 & 0.071 & 0 & 0 & 0.027 & 0 & 0 & 0.002 & 0 & 0 & 0.1 \\
\hline \multicolumn{12}{|c|}{ GACA source } \\
\hline \multirow[t]{3}{*}{ Season } & Year & na & $\mathrm{BB}$ & $\mathrm{DD}$ & $\mathrm{BIO}$ & URB & AGED & VOG & SS & $X X$ & total \\
\hline & 2007 & 0.019 & 0 & 0 & 0 & 0.092 & 0 & 0 & 0 & 0 & 0.111 \\
\hline & 2008 & 0.049 & 0 & 0 & 0 & 0.088 & 0 & 0 & 0 & 0 & 0.137 \\
\hline \multirow[t]{5}{*}{ DJF } & 2009 & 0.012 & 0 & 0 & 0 & 0.125 & 0 & 0 & 0 & 0 & 0.137 \\
\hline & 2010 & 0.07 & 0 & 0 & 0 & 0.069 & 0 & 0 & 0 & 0 & 0.139 \\
\hline & 2011 & 0.101 & 0 & 0 & 0 & 0 & 0 & 0 & 0 & 0 & 0.101 \\
\hline & 2007 & 0 & 0 & 0 & 0 & 0.191 & 0.015 & 0 & 0 & 0 & 0.206 \\
\hline & 2008 & 0.006 & 0 & 0 & 0 & 0.155 & 0 & 0 & 0 & 0 & 0.161 \\
\hline \multirow[t]{5}{*}{ MAM } & 2009 & 0.01 & 0 & 0 & 0 & 0.191 & 0 & 0 & 0 & 0 & 0.201 \\
\hline & 2010 & 0.001 & 0 & 0 & 0 & 0.162 & 0 & 0 & 0 & 0 & 0.163 \\
\hline & 2011 & 0 & 0 & 0 & 0 & 0.193 & 0 & 0 & 0 & 0 & 0.193 \\
\hline & 2007 & 0.003 & 0 & 0 & 0 & 0.216 & 0 & 0 & 0 & 0 & 0.219 \\
\hline & 2008 & 0.007 & 0 & 0 & 0 & 0.171 & 0 & 0 & 0 & 0 & 0.178 \\
\hline \multirow[t]{5}{*}{$\mathrm{JJA}$} & 2009 & 0.003 & 0 & 0 & 0 & 0.187 & 0 & 0 & 0 & 0 & 0.19 \\
\hline & 2010 & 0.004 & 0 & 0 & 0 & 0.15 & 0 & 0 & 0 & 0 & 0.154 \\
\hline & 2011 & 0.009 & 0 & 0 & 0 & 0.163 & 0 & 0 & 0 & 0 & 0.172 \\
\hline & 2007 & 0.003 & 0 & 0 & 0 & 0.125 & 0 & 0 & 0 & 0 & 0.128 \\
\hline & 2008 & 0.015 & 0 & 0 & 0 & 0.13 & 0 & 0 & 0 & 0 & 0.145 \\
\hline \multirow[t]{3}{*}{ SON } & 2009 & 0.007 & 0 & 0 & 0 & 0.137 & 0 & 0 & 0 & 0 & 0.144 \\
\hline & 2010 & 0.009 & 0 & 0 & 0 & 0.076 & 0 & 0 & 0 & 0 & 0.085 \\
\hline & 2011 & 0.002 & 0 & 0 & 0 & 0.097 & 0 & 0 & 0 & 0 & 0.099 \\
\hline
\end{tabular}


Table S4b. AOD assigned to each aerosol component by MACC (abbreviations explained in Table 1 in the manuscript) for the region in Northwest Europe (48-53 N $\left./ 3-8^{\circ} \mathrm{E}\right)$. Seasons: DJF: DecemberFebruary, MAM: March-May, JJA: June-August, SON: September-November.

\begin{tabular}{|l|r|r|r|r|r|r|r|}
\hline Season & \multicolumn{1}{l|}{ Year } & BC & \multicolumn{1}{l|}{ DD } & \multicolumn{1}{l|}{ OC } & \multicolumn{1}{l|}{ SO4 } & \multicolumn{1}{l|}{ SS } & \multicolumn{1}{l|}{ total } \\
\hline & 2007 & 0.004 & 0.01 & 0.005 & 0.028 & 0.131 & 0.177 \\
\hline & 2008 & 0.008 & 0.017 & 0.009 & 0.053 & 0.062 & 0.149 \\
\hline DJF & 2009 & 0.004 & 0.015 & 0.006 & 0.044 & 0.057 & 0.126 \\
\hline & 2010 & 0.004 & 0.013 & 0.01 & 0.042 & 0.073 & 0.141 \\
\hline & 2011 & 0.005 & 0.015 & 0.008 & 0.057 & 0.073 & 0.158 \\
\hline & 2007 & 0.01 & 0.032 & 0.012 & 0.113 & 0.035 & 0.202 \\
\hline & 2008 & 0.008 & 0.048 & 0.01 & 0.103 & 0.057 & 0.226 \\
\hline MAM & 2009 & 0.008 & 0.043 & 0.013 & 0.11 & 0.043 & 0.216 \\
\hline & 2010 & 0.008 & 0.034 & 0.011 & 0.106 & 0.038 & 0.197 \\
\hline & 2011 & 0.01 & 0.042 & 0.015 & 0.127 & 0.034 & 0.228 \\
\hline & 2007 & 0.009 & 0.034 & 0.02 & 0.124 & 0.028 & 0.215 \\
\hline & 2008 & 0.009 & 0.043 & 0.022 & 0.105 & 0.033 & 0.211 \\
\hline JJA & 2009 & 0.008 & 0.045 & 0.018 & 0.111 & 0.033 & 0.215 \\
\hline & 2010 & 0.009 & 0.034 & 0.026 & 0.109 & 0.03 & 0.208 \\
\hline & 2011 & 0.008 & 0.046 & 0.019 & 0.101 & 0.038 & 0.212 \\
\hline & 2007 & 0.006 & 0.024 & 0.009 & 0.068 & 0.046 & 0.151 \\
\hline & 2008 & 0.005 & 0.036 & 0.008 & 0.065 & 0.048 & 0.162 \\
\hline SON & 2009 & 0.007 & 0.03 & 0.01 & 0.074 & 0.065 & 0.187 \\
\hline & 2010 & 0.006 & 0.021 & 0.009 & 0.064 & 0.043 & 0.143 \\
\hline & 2011 & 0.007 & 0.034 & 0.01 & 0.075 & 0.041 & 0.167 \\
\hline & & & & & & & \\
\hline
\end{tabular}


Table S5a. AOD assigned to each aerosol type and source by GACA (abbreviations explained in Table 1 in the manuscript) for the region in Thailand (15-20 N/100-105 E). Seasons: DJF: DecemberFebruary, MAM: March-May, JJA: June-August, SON: September-November.

\begin{tabular}{|c|c|c|c|c|c|c|c|c|c|c|c|}
\hline \multicolumn{12}{|c|}{ GACA type } \\
\hline \multirow[t]{3}{*}{ Season } & Year & SNA & SN & SA & MNA & $\mathrm{MN}$ & $\mathrm{MA}$ & LNA & LN & LA & total \\
\hline & 2007 & 0.024 & 0.038 & 0.033 & 0.012 & 0.059 & 0.052 & 0.021 & 0.035 & 0.016 & 0.29 \\
\hline & 2008 & 0.053 & 0.037 & 0 & 0.036 & 0.035 & 0 & 0.035 & 0.035 & 0 & 0.231 \\
\hline \multirow[t]{5}{*}{ DJF } & 2009 & 0.034 & 0.04 & 0 & 0.026 & 0.082 & 0.025 & 0.025 & 0.035 & 0 & 0.267 \\
\hline & 2010 & 0.1 & 0.06 & 0 & 0.052 & 0 & 0 & 0.025 & 0 & 0 & 0.237 \\
\hline & 2011 & 0.07 & 0.002 & 0 & 0.061 & 0.027 & 0 & 0.039 & 0.006 & 0 & 0.205 \\
\hline & 2007 & 0.083 & 0.06 & 0.162 & 0.047 & 0.046 & 0.078 & 0.023 & 0 & 0 & 0.499 \\
\hline & 2008 & 0.086 & 0.138 & 0.047 & 0.051 & 0.084 & 0 & 0.056 & 0.022 & 0 & 0.484 \\
\hline \multirow{5}{*}{ MAM } & 2009 & 0.085 & 0.086 & 0.056 & 0.081 & 0.099 & 0.051 & 0.034 & 0 & 0 & 0.492 \\
\hline & 2010 & 0.058 & 0.07 & 0.207 & 0.063 & 0.109 & 0.046 & 0.028 & 0.016 & 0 & 0.597 \\
\hline & 2011 & 0.249 & 0.008 & 0 & 0.163 & 0.011 & 0 & 0.033 & 0 & 0 & 0.464 \\
\hline & 2007 & 0.079 & 0 & 0 & 0.068 & 0 & 0 & 0.115 & 0 & 0 & 0.262 \\
\hline & 2008 & 0.061 & 0 & 0 & 0.069 & 0 & 0 & 0.166 & 0 & 0 & 0.296 \\
\hline \multirow[t]{5}{*}{ JJA } & 2009 & 0.057 & 0 & 0 & 0.071 & 0 & 0 & 0.125 & 0 & 0 & 0.253 \\
\hline & 2010 & 0.053 & 0 & 0 & 0.095 & 0 & 0 & 0.118 & 0.006 & 0 & 0.272 \\
\hline & 2011 & 0.078 & 0 & 0 & 0.09 & 0 & 0 & 0.125 & 0 & 0 & 0.293 \\
\hline & 2007 & 0.135 & 0 & 0 & 0.172 & 0 & 0 & 0.035 & 0 & 0 & 0.342 \\
\hline & 2008 & 0.077 & 0.001 & 0 & 0.059 & 0 & 0 & 0.085 & 0 & 0 & 0.222 \\
\hline \multirow[t]{3}{*}{ SON } & 2009 & 0.098 & 0 & 0 & 0.114 & 0 & 0 & 0.036 & 0 & 0 & 0.248 \\
\hline & 2010 & 0.067 & 0 & 0 & 0.079 & 0 & 0 & 0.084 & 0 & 0 & 0.23 \\
\hline & 2011 & 0.098 & 0 & 0 & 0.075 & 0 & 0 & 0.068 & 0 & 0 & 0.241 \\
\hline \multicolumn{12}{|c|}{ GACA source } \\
\hline \multirow[t]{3}{*}{ Season } & Year & na & $\mathrm{BB}$ & $\mathrm{DD}$ & $\mathrm{BIO}$ & URB & AGED & VOG & SS & $X X$ & total \\
\hline & 2007 & 0.016 & 0.181 & 0 & 0 & 0.068 & 0.024 & 0 & 0 & 0 & 0.289 \\
\hline & 2008 & 0 & 0 & 0 & 0.053 & 0.178 & 0 & 0 & 0 & 0 & 0.231 \\
\hline \multirow[t]{5}{*}{ DJF } & 2009 & 0.025 & 0.122 & 0 & 0 & 0.12 & 0 & 0 & 0 & 0 & 0.267 \\
\hline & 2010 & 0 & 0 & 0 & 0.1 & 0.138 & 0 & 0 & 0 & 0 & 0.238 \\
\hline & 2011 & 0.008 & 0 & 0 & 0.07 & 0.128 & 0 & 0 & 0 & 0 & 0.206 \\
\hline & 2007 & 0 & 0.24 & 0 & 0.083 & 0.176 & 0 & 0 & 0 & 0 & 0.499 \\
\hline & 2008 & 0.022 & 0.047 & 0 & 0.086 & 0.328 & 0 & 0 & 0 & 0 & 0.483 \\
\hline \multirow[t]{5}{*}{ MAM } & 2009 & 0 & 0.107 & 0 & 0.085 & 0.299 & 0 & 0 & 0 & 0 & 0.491 \\
\hline & 2010 & 0.062 & 0.207 & 0 & 0.058 & 0.27 & 0 & 0 & 0 & 0 & 0.597 \\
\hline & 2011 & 0.052 & 0 & 0 & 0.249 & 0.163 & 0 & 0 & 0 & 0 & 0.464 \\
\hline & 2007 & 0 & 0 & 0 & 0.079 & 0.184 & 0 & 0 & 0 & 0 & 0.263 \\
\hline & 2008 & 0 & 0 & 0 & 0 & 0.296 & 0 & 0 & 0 & 0 & 0.296 \\
\hline \multirow[t]{5}{*}{ JJA } & 2009 & 0 & 0 & 0 & 0.057 & 0.195 & 0 & 0 & 0 & 0 & 0.252 \\
\hline & 2010 & 0.006 & 0 & 0 & 0.053 & 0.213 & 0 & 0 & 0 & 0 & 0.272 \\
\hline & 2011 & 0 & 0 & 0 & 0.078 & 0.215 & 0 & 0 & 0 & 0 & 0.293 \\
\hline & 2007 & 0 & 0 & 0 & 0 & 0.342 & 0 & 0 & 0 & 0 & 0.342 \\
\hline & 2008 & 0.001 & 0 & 0 & 0.077 & 0.144 & 0 & 0 & 0 & 0 & 0.222 \\
\hline \multirow[t]{3}{*}{ SON } & 2009 & 0 & 0 & 0 & 0.098 & 0.15 & 0 & 0 & 0 & 0 & 0.248 \\
\hline & 2010 & 0 & 0 & 0 & 0.067 & 0.162 & 0 & 0 & 0 & 0 & 0.229 \\
\hline & 2011 & 0 & 0 & 0 & 0.098 & 0.143 & 0 & 0 & 0 & 0 & 0.241 \\
\hline
\end{tabular}


Table S5b. AOD assigned to each aerosol component by MACC (abbreviations explained in Table 1 in the manuscript) for the region in Thailand (15-20 $\left.\mathrm{N} / 100-105^{\circ} \mathrm{E}\right)$. Seasons: DJF: December-February, MAM: March-May, JJA: June-August, SON: September-November.

\begin{tabular}{|l|r|r|r|r|r|r|r|}
\hline Season & Year & BC & \multicolumn{1}{l|}{ DD } & \multicolumn{1}{l|}{ OC } & \multicolumn{1}{l}{ SO4 } & \multicolumn{1}{l|}{ SS } & total \\
\hline & 2007 & 0.017 & 0.064 & 0.082 & 0.107 & 0.042 & 0.313 \\
\hline & 2008 & 0.015 & 0.063 & 0.052 & 0.126 & 0.027 & 0.282 \\
\hline DJF & 2009 & 0.022 & 0.09 & 0.089 & 0.14 & 0.052 & 0.392 \\
\hline & 2010 & 0.017 & 0.066 & 0.065 & 0.116 & 0.033 & 0.297 \\
\hline & 2011 & 0.018 & 0.067 & 0.074 & 0.142 & 0.035 & 0.336 \\
\hline & 2007 & 0.025 & 0.106 & 0.127 & 0.149 & 0.049 & 0.455 \\
\hline & 2008 & 0.02 & 0.14 & 0.09 & 0.184 & 0.061 & 0.495 \\
\hline MAM & 2009 & 0.023 & 0.118 & 0.12 & 0.13 & 0.043 & 0.434 \\
\hline & 2010 & 0.033 & 0.161 & 0.159 & 0.178 & 0.055 & 0.585 \\
\hline & 2011 & 0.021 & 0.102 & 0.134 & 0.176 & 0.052 & 0.485 \\
\hline & 2007 & 0.007 & 0.052 & 0.046 & 0.106 & 0.056 & 0.268 \\
\hline & 2008 & 0.007 & 0.084 & 0.042 & 0.098 & 0.06 & 0.29 \\
\hline JJA & 2009 & 0.006 & 0.071 & 0.039 & 0.093 & 0.051 & 0.261 \\
\hline & 2010 & 0.008 & 0.059 & 0.047 & 0.094 & 0.06 & 0.268 \\
\hline & 2011 & 0.007 & 0.076 & 0.046 & 0.103 & 0.067 & 0.299 \\
\hline & 2007 & 0.013 & 0.05 & 0.048 & 0.185 & 0.039 & 0.334 \\
\hline & 2008 & 0.008 & 0.044 & 0.042 & 0.121 & 0.041 & 0.257 \\
\hline SON & 2009 & 0.011 & 0.046 & 0.046 & 0.148 & 0.032 & 0.282 \\
\hline & 2010 & 0.011 & 0.041 & 0.049 & 0.154 & 0.042 & 0.297 \\
\hline & 2011 & 0.009 & 0.046 & 0.044 & 0.13 & 0.038 & 0.267 \\
\hline
\end{tabular}


Table S6a. AOD assigned to each aerosol type and source by GACA (abbreviations explained in Table 1 in the manuscript) for the region in Northeast China $\left(35-40^{\circ} \mathrm{N} / 115-120^{\circ} \mathrm{E}\right)$. Seasons: DJF: December-February, MAM: March-May, JJA: June-August, SON: September-November.

\begin{tabular}{|c|c|c|c|c|c|c|c|c|c|c|c|}
\hline \multicolumn{12}{|c|}{ GACA type } \\
\hline \multirow[t]{3}{*}{ Season } & Year & SNA & SN & SA & MNA & $\mathrm{MN}$ & $\mathrm{MA}$ & LNA & LN & LA & total \\
\hline & 2007 & 0 & 0.029 & 0.02 & 0 & 0.03 & 0.095 & 0 & 0.054 & 0.25 & 0.478 \\
\hline & 2008 & 0 & 0.079 & 0.046 & 0 & 0.027 & 0.012 & 0 & 0.109 & 0.209 & 0.482 \\
\hline \multirow[t]{5}{*}{ DJF } & 2009 & 0 & 0.014 & 0 & 0 & 0.052 & 0.06 & 0 & 0.038 & 0.365 & 0.529 \\
\hline & 2010 & 0 & 0.091 & 0.025 & 0 & 0.041 & 0.048 & 0 & 0.162 & 0.147 & 0.514 \\
\hline & 2011 & 0.008 & 0.061 & 0.038 & 0 & 0.052 & 0 & 0 & 0.188 & 0.139 & 0.486 \\
\hline & 2007 & 0 & 0.02 & 0.018 & 0 & 0.263 & 0.019 & 0 & 0.242 & 0.274 & 0.836 \\
\hline & 2008 & 0 & 0.019 & 0 & 0 & 0.261 & 0.291 & 0 & 0.117 & 0.219 & 0.907 \\
\hline \multirow{5}{*}{ MAM } & 2009 & 0 & 0.032 & 0 & 0.024 & 0.282 & 0.009 & 0.01 & 0.235 & 0.141 & 0.733 \\
\hline & 2010 & 0.016 & 0.052 & 0 & 0.037 & 0.163 & 0 & 0 & 0.433 & 0.01 & 0.711 \\
\hline & 2011 & 0 & 0 & 0 & 0 & 0.122 & 0 & 0 & 0.591 & 0 & 0.713 \\
\hline & 2007 & 0.401 & 0.014 & 0.012 & 0.115 & 0.191 & 0.197 & 0 & 0 & 0 & 0.93 \\
\hline & 2008 & 0.423 & 0 & 0 & 0.127 & 0.362 & 0 & 0 & 0.023 & 0 & 0.935 \\
\hline \multirow[t]{5}{*}{ JJA } & 2009 & 0.178 & 0.02 & 0 & 0.235 & 0.101 & 0 & 0 & 0.082 & 0 & 0.616 \\
\hline & 2010 & 0.425 & 0 & 0 & 0.575 & 0 & 0 & 0 & 0 & 0 & 1 \\
\hline & 2011 & 0.434 & 0 & 0 & 0.431 & 0.05 & 0 & 0.045 & 0 & 0 & 0.96 \\
\hline & 2007 & 0.112 & 0.061 & 0 & 0.07 & 0.138 & 0.019 & 0 & 0.074 & 0.028 & 0.502 \\
\hline & 2008 & 0.135 & 0.075 & 0 & 0.063 & 0.142 & 0 & 0 & 0.108 & 0 & 0.523 \\
\hline \multirow[t]{3}{*}{ SON } & 2009 & 0.05 & 0.066 & 0 & 0.127 & 0.155 & 0 & 0 & 0.179 & 0 & 0.577 \\
\hline & 2010 & 0.16 & 0.025 & 0 & 0.132 & 0.107 & 0 & 0.007 & 0.126 & 0.005 & 0.562 \\
\hline & 2011 & 0.115 & 0.038 & 0 & 0.25 & 0.16 & 0 & 0.005 & 0 & 0 & 0.568 \\
\hline \multicolumn{12}{|c|}{ GACA source } \\
\hline \multirow[t]{3}{*}{ Season } & Year & na & $\mathrm{BB}$ & $\mathrm{DD}$ & $\mathrm{BIO}$ & URB & AGED & VOG & SS & $X X$ & total \\
\hline & 2007 & 0.079 & 0.345 & 0 & 0 & 0.054 & 0 & 0 & 0 & 0 & 0.478 \\
\hline & 2008 & 0.039 & 0.255 & 0 & 0 & 0.188 & 0 & 0 & 0 & 0 & 0.482 \\
\hline \multirow[t]{5}{*}{ DJF } & 2009 & 0.014 & 0.425 & 0 & 0 & 0.09 & 0 & 0 & 0 & 0 & 0.529 \\
\hline & 2010 & 0.073 & 0.239 & 0 & 0 & 0.203 & 0 & 0 & 0 & 0 & 0.515 \\
\hline & 2011 & 0.008 & 0.177 & 0 & 0 & 0.301 & 0 & 0 & 0 & 0 & 0.486 \\
\hline & 2007 & 0.057 & 0.274 & 0 & 0 & 0.505 & 0 & 0 & 0 & 0 & 0.836 \\
\hline & 2008 & 0.019 & 0.51 & 0 & 0 & 0.378 & 0 & 0 & 0 & 0 & 0.907 \\
\hline \multirow[t]{5}{*}{ MAM } & 2009 & 0.076 & 0.141 & 0 & 0 & 0.517 & 0 & 0 & 0 & 0 & 0.734 \\
\hline & 2010 & 0.115 & 0 & 0 & 0 & 0.596 & 0 & 0 & 0 & 0 & 0.711 \\
\hline & 2011 & 0 & 0 & 0 & 0 & 0.713 & 0 & 0 & 0 & 0 & 0.713 \\
\hline & 2007 & 0.026 & 0.197 & 0 & 0 & 0.708 & 0 & 0 & 0 & 0 & 0.931 \\
\hline & 2008 & 0.023 & 0 & 0 & 0 & 0.912 & 0 & 0 & 0 & 0 & 0.935 \\
\hline \multirow[t]{5}{*}{ JJA } & 2009 & 0.02 & 0 & 0 & 0 & 0.596 & 0 & 0 & 0 & 0 & 0.616 \\
\hline & 2010 & 0 & 0 & 0 & 0 & 1 & 0 & 0 & 0 & 0 & 1 \\
\hline & 2011 & 0.096 & 0 & 0 & 0 & 0.865 & 0 & 0 & 0 & 0 & 0.961 \\
\hline & 2007 & 0.047 & 0 & 0 & 0 & 0.456 & 0 & 0 & 0 & 0 & 0.503 \\
\hline & 2008 & 0 & 0 & 0 & 0 & 0.524 & 0 & 0 & 0 & 0 & 0.524 \\
\hline \multirow[t]{3}{*}{ SON } & 2009 & 0 & 0.066 & 0 & 0 & 0.511 & 0 & 0 & 0 & 0 & 0.577 \\
\hline & 2010 & 0.037 & 0 & 0 & 0 & 0.525 & 0 & 0 & 0 & 0 & 0.562 \\
\hline & 2011 & 0.043 & 0 & 0 & 0 & 0.525 & 0 & 0 & 0 & 0 & 0.568 \\
\hline
\end{tabular}


Table S6b. AOD assigned to each aerosol component by MACC (abbreviations explained in Table 1 in

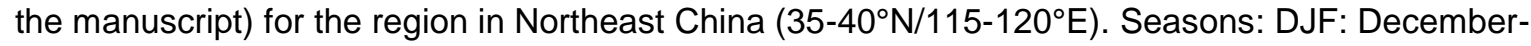
February, MAM: March-May, JJA: June-August, SON: September-November.

\begin{tabular}{|l|r|r|l|l|r|r|r|}
\hline Season & Year & \multicolumn{1}{l|}{ BC } & DD & \multicolumn{1}{l|}{ OC } & \multicolumn{1}{l|}{ SO4 } & \multicolumn{1}{l|}{ SS } & total \\
\hline & 2007 & 0.031 & 0.135 & 0.051 & 0.31 & 0.024 & 0.551 \\
\hline & 2008 & 0.026 & 0.129 & 0.023 & 0.16 & 0.012 & 0.35 \\
\hline DJF & 2009 & 0.037 & 0.147 & 0.031 & 0.239 & 0.015 & 0.469 \\
\hline & 2010 & 0.03 & 0.119 & 0.061 & 0.384 & 0.026 & 0.621 \\
\hline & 2011 & 0.035 & 0.115 & 0.041 & 0.288 & 0.021 & 0.5 \\
\hline & 2007 & 0.04 & 0.206 & 0.057 & 0.428 & 0.02 & 0.751 \\
\hline & 2008 & 0.041 & 0.314 & 0.068 & 0.425 & 0.027 & 0.876 \\
\hline MAM & 2009 & 0.035 & 0.234 & 0.061 & 0.328 & 0.018 & 0.676 \\
\hline & 2010 & 0.032 & 0.237 & 0.054 & 0.348 & 0.022 & 0.693 \\
\hline & 2011 & 0.034 & 0.237 & 0.055 & 0.282 & 0.02 & 0.629 \\
\hline & 2007 & 0.038 & 0.155 & 0.077 & 0.432 & 0.022 & 0.725 \\
\hline & 2008 & 0.037 & 0.211 & 0.074 & 0.456 & 0.029 & 0.807 \\
\hline JJA & 2009 & 0.035 & 0.212 & 0.074 & 0.39 & 0.02 & 0.732 \\
\hline & 2010 & 0.043 & 0.205 & 0.089 & 0.513 & 0.028 & 0.878 \\
\hline & 2011 & 0.045 & 0.239 & 0.099 & 0.535 & 0.032 & 0.95 \\
\hline & 2007 & 0.035 & 0.154 & 0.05 & 0.348 & 0.014 & 0.602 \\
\hline & 2008 & 0.03 & 0.184 & 0.038 & 0.261 & 0.012 & 0.525 \\
\hline SON & 2009 & 0.032 & 0.184 & 0.046 & 0.279 & 0.014 & 0.555 \\
\hline & 2010 & 0.032 & 0.153 & 0.047 & 0.31 & 0.014 & 0.556 \\
\hline & 2011 & 0.03 & 0.185 & 0.053 & 0.356 & 0.02 & 0.644 \\
\hline
\end{tabular}

